\title{
Editorial
}

\section{Special issue of Wave Motion - "Nonlinear waves in solids: in memory of Alexander M. Samsonov"}

This special issue of Wave Motion is dedicated to the memory of Professor Alexander M. Samsonov (30.09.1948 - 07.10.2017). Our aim is to present recent research in the area of "Nonlinear Waves in Solids", where Professor Samsonov has made many pioneering contributions.

Alexander Samsonov was born on September 30, 1948 in St. Petersburg, Russia. He graduated from the Physics and Mechanics School of the Leningrad Polytechnic Institute (now the Peter the Great Saint Petersburg Polytechnic University) in 1972. Alexander obtained his PhD degree in 1979 for his work in Optimal Control Theory under the supervision of Prof. K.A. Lurie. In 2002 Alexander was awarded a Doctor of Science degree in Theoretical Physics for studies on the subject of "Nonlinear elastic waves theory for solid waveguides" in the Ioffe Institute of the Russian Academy of Sciences. He worked in this famous Institute from 1979, going through the ranks from a Junior Research Fellow to the Head of the Solid State Theory Division. From 2005 Alexander was also a Professor of Applied Mathematics at the Peter the Great St. Petersburg Polytechnic University.

Throughout his career Alexander's main research was in the area of nonlinear bulk strain solitary waves in solid waveguides. Alexander has initiated and developed, together with his students and collaborators, a new research direction which, eventually, formed the basis for the book [1]. His work included the development of mathematical models of Boussinesq type describing long nonlinear longitudinal bulk strain waves in various elastic waveguides (e.g., rods, plates, shells, etc.) within the scope of the Murnaghan model of nonlinear dynamic elasticity, and application of the derived model equations to the description of solitary waves and their evolution in various non-homogeneous waveguides (e.g. rods of variable cross-section, layered waveguides, etc.) using analytical approaches and direct numerical simulations. Alexander has inspired and guided some invaluable and detailed experimental work carried out in the Ioffe Institute. The experimental group originally included Yury Ostrovsky, Galina Dreiden and Irina Semenova. Together, they worked on the generation and observation of longitudinal bulk strain solitary waves in various solid waveguides.

Alexander had very broad research interests. In particular, he worked on application of methods of nonlinear mathematical physics to computational and systems biology, including, but not limited to, modelling of gene networks controlling early Drosophila embryogenesis [2], mechanistic aspect of molecular transport [3], as well as of the microRNA regulatory role in cells [4].

Alexander was a passionate and inspiring teacher for his students and younger colleagues, dedicating much of his time to their training, and challenging them to think creatively and independently. Many of these young scientists now also work on various waves-related problems. Alexander's ideas continue to influence researchers working in the area of nonlinear waves in solids.

Alexander was a very optimistic person loving travel, literature, photography, fine arts and music, of which German and Italian operas were his favourites. We will always remember him as a wonderful colleague and friend. We hope that this special issue will serve both as a reference for those who are already actively working on nonlinear waves in solids, and will help to engage a new generation of researchers.

The special issue includes 11 papers devoted to various aspects of nonlinear waves in solids, including analytical, numerical and experimental developments.

The paper by A. Pau and F. Vestroni [5] belongs to the classical area of acousto-elasticity, and is devoted to a detailed study of the individual effects of material and geometric nonlinearities on the changes in shear and longitudinal speeds of bulk waves, and their polarization as a function of the initial pre-stress. Three types of pre-stress are considered: hydrostatic, biaxial and uniaxial pre-stress. It is shown that material nonlinearity has a significant effect on the amount of speed change, and that pre-stress can lead to anisotropic behaviour and coupling between shear and pressure waves.

The derivations of model equations describing long weakly nonlinear longitudinal bulk strain waves in elastic rods are revisited within the scope of the Murnaghan model in the paper by F.E. Garbuzov et al. [6]. Two forced Boussinesq-type models are derived for a circular rod with axially symmetric loading and longitudinal pre-stretch from the full problem formulation using the presence of small parameters. Basic dynamical properties of the models are compared, and the equations are suggested as models for the related experimental research.

Soliton solutions of a Boussinesq-type equation with displacement-type nonlinearities linked to the HeimburgJackson model describing longitudinal waves (density charge) in biomembranes are investigated in the paper by T. Peets et al. [7]. The possibility of the existence of a soliton doublet, an ensemble of two co-existing 
solitary waves moving with the same velocities but different amplitudes, is shown using phase plane analysis and numerical simulations.

M.R. Tranter considers the propagation of a solitary wave in non-homogeneous layered bars described by a system of coupled Boussinesq-type equations with piecewise-constant coefficients subject to continuity of displacement and stress on the boundaries with the help of numerical simulations [8]. The numerical scheme is applied to describe the transmission and reflection of longitudinal waves in layered waveguides with delamination, extending previous studies.

Numerical simulations are also used in the paper by A. Salupere and M. Rätsep [9] to study the formation and interaction of solitary waves within the scope of the so-called hyperelastic rod equation, earlier suggested as a model for waves with moderate wavelength. The model constitutes a modification of the Korteweg - de Vries equation and contains three arbitrary parameters. The authors are particularly concerned with the effect of these parameters on the nature of solutions.

The paper by O.V. Rudenko and C.M. Hedberg [10] is devoted to nonlinear waves in a lattice with holonomic constraints: atoms can move only along parallel lines. The dynamics of the lattice is studied in the continuum approximation, and a new equation is derived to describe the waves. It is shown that the structure exhibits anisotropic properties similar to a skeletal muscle with stretched fibres. Particular solitary wave solutions of the derived equation are studied in detail.

Nonlinear waves in a lattice are also studied in the paper by A.V. Porubov and A.E. Osokina [11]. Plane longitudinal waves in a 2D graphene-type hexagonal lattice are considered in the continuum approximation, and a Boussinesq-type equation similar to the equation describing longitudinal waves in a rod is obtained. The model is geometrically linear, but includes nonlinear terms in the potentials of interactions between the particles of the lattice.

Propagation of elastic waves in damaged media is modelled by $\mathrm{H}$. Berjamin et al. [12]. A constitutive model combines Murnaghan model for hyperelasticity with an additional evolution of a scalar variable characterising the long-time softening of the material. The equations of motion are cast into the form of a nonlinear hyperbolic system of conservation laws and solved numerically using a finite-volume method. In particular, the generation of solitary waves in a periodic layered medium is described.

The paper by R. De Pascalis et al. [13] is devoted to the modelling of the shearing motions within the quasi-linear viscoelastic model widely used to investigate processes in soft tissues and bio-materials. Kink-type solitary waves are studied with the help of the derived nonlinear second-order ordinary differential equation. It is shown, for the stress relaxation function in the form of a Prony series, that kinks can exist for strongly elliptic strain energy, but not for the Mooney-Rivlin model. The Yeoh model is studied using numerical simulations.

P. Zhu and H.-H. Dai consider a 3D internal-variable formulation for a propagating phase boundary in a shape memory alloy bar in order to obtain an extra admissibility condition for the solution of the problem [14]. The derived condition is then used in the study of an impact problem, and the unique solution is constructed analytically for all possible values of the impact velocity. Comparison is made with the results obtained using the maximal dissipation rate criterion.

A.T. Il'ichev and V.Ja. Tomashpolskii [15] study the effect of a pre-stressed ice cover on the evolution of nonlinear surface envelope waves. The ice cover is modelled as a Kirchhoff-Love plate of considerable thickness. The fluid motion is described by the two-dimensional Euler equations including the additional pressure from the freely floating plate. The envelope solitary waves are studied in detail. The characteristic values of the wavelength and wave velocity are determined depending on the fluid depth and ice thickness.

\section{References}

[1] A.M. Samsonov, Strain solitons in solids and how to construct them, Chapman \& Hall/CRC, 2001.

[2] V.V. Gursky, K.N. Kozlov, A.M. Samsonov, and J. Reinitz, Cell divisions as a mechanism for selection in stable steady states of multi-stationary gene circuits, Physica D 218 (2006) 70-76.

[3] S.A. Rukolaine, A.M. Samsonov, Local immobilization of particles in mass transfer described by a Jeffreystype equation, Phys. Rev. E 88 (2013) 062116.

[4] M.A. Duk, M.G. Samsonova and A.M. Samsonov, Dynamics of miRNA-driven feed-forward loop depends upon miRNA action mechanisms, BMC Genomics 15 (Suppl. 12) (2014) S9. 
[5] A. Pau, F. Vestroni, The role of material and geometric nonlinearities in acoustoelasticity, Wave Motion 86 (2019) 79-90.

[6] F.E. Garbuzov, K.R. Khusnutdinova, I.V. Semenova, On Boussinesq-type models for long longitudinal waves in elastic rods, Wave Motion 88 (2019) 129-143.

[7] T. Peets, K. Tamm, P. Simson, J. Engelbrecht, On solutions of a Boussinesq-type equation with displacement-dependent nonlinearity: a soliton doublet, Wave Motion 85 (2019) 10-17.

[8] M.R. Tranter, Solitary wave propagation in elastic bars with multiple sections and layers, Wave Motion 86 (2019) 21-31.

[9] A. Salupere, M. Rätsep, On solitonic solutions for the hyperelastic rod equation, Wave Motion ?? (2019) ??

[10] O.V. Rudenko, C.M. Hedberg, Strong nonlinearity, anisotropy, and solitons in a lattice with holonomic constraints, Wave Motion 89 (2019) 104-115.

[11] A.V. Porubov, A.E. Osokina, Double dispersion equation for nonlinear waves in a graphene-type hexagonal lattice, Wave Motion 89 (2019) 185-192.

[12] H. Berjamin, B. Lombard, G. Chiavassa, N. Favrie, Plane-strain waves in nonlinear elastic solids with softening, Wave Motion 89 (2019) 65-78.

[13] R. De Pascalis, G. Napoli, G. Saccomandi, Kink-type solitary waves within the quasi-linear viscoelastic model, Wave Motion 86 (2019) 195-202.

[14] P. Zhu, H.-H. Dai, On the derivation of an extra admissibility condition for phase boundary propagation in an SMA bar based on a 3-D formulation, Wave Motion ?? (2019) ??

[15] A.T. Il'ichev, V.Ja. Tomashpolskii, Characteristic parameters of nonlinear surface envelope waves beneath an ice cover under pre-stress, Wave Motion 86 (2019) 11-20.

Guest Editors

Dr. Karima Khusnutdinova*

Department of Mathematical Sciences, Loughborough University,

Loughborough LE11 3TU, United Kingdom

E-mail address: K.Khusnutdinova@lboro.ac.uk

Prof. Yibin Fu

School of Computing and Mathematics, Keele University,

Staffordshire ST5 5BG, United Kingdom

E-mail address: y.fu@keele.ac.uk

Prof. Roger Grimshaw

Department of Mathematics, University College London,

London WC1E 6BT, United Kingdom

E-mail address: r.grimshaw@ucl.ac.uk

and

Prof. Franco Pastrone

Accademia delle Scienze di Torino, Via Maria Vittoria 4, 10123, Torino, Italy

E-mail address: franco.pastrone@unito.it

* Corresponding author. Tel. +44 1509228202 